\title{
Pre-treatment circulating neutrophil count is an independent prognostic factor in oropharyngeal cancer
}

\author{
Liang $\mathrm{Li}^{1,2 \#}$, Guangping Liu ${ }^{2 \#}$, Honglue $\mathrm{Lu}^{1,3 \#}$, Kai Jin ${ }^{1}$, Xiang Zhai ${ }^{1}$, Mengqian Zhou ${ }^{1}$, Yuansheng Duan ${ }^{1}$, \\ Kai Yue ${ }^{1}$, Yansheng $\mathrm{Wu}^{1}$, Xudong Wang ${ }^{1}$ \\ ${ }^{1}$ Department of Maxillofacial and E.N.T. Oncology, Tianjin Medical University Cancer Institute and Hospital, National Clinical Research Center \\ for Cancer, Key Laboratory of Cancer Prevention and Therapy, Tianjin's Clinical Research Center for Cancer, Tianjin, China; ${ }^{2}$ Department of \\ Otolaryngology, Tianjin Children's Hospital, Tianjin University Children's Hospital, Tianjin, China; ${ }^{3}$ Department of Otolaryngology, the Affiliated \\ Hospital of Chengde Medical College, Chengde, China \\ Contributions: (I) Conception and design: L Li; (II) Administrative support: X Wang; (III) Provision of study materials or patients: H Lu; (IV) \\ Collection and assembly of data: G Liu; (V) Data analysis and interpretation: L Li; (VI) Manuscript writing: All authors; (VII) Final approval of \\ manuscript: All authors. \\ "These authors contributed equally to this work. \\ Correspondence to: Prof. Xudong Wang; Yansheng Wu. Department of Maxillofacial and E.N.T. Oncology, Tianjin Medical University Cancer \\ Institute and Hospital, National Clinical Research Center for Cancer, Key Laboratory of Cancer Prevention and Therapy, Tianjin's Clinical Research \\ Center for Cancer, Tianjin, China. Email: WXD.1133@163.com; yansheng1981@163.com.
}

Background: Oropharyngeal cancer (OPC) is a type of head and neck squamous cell cancer, the incidence
of which has increased in recent years. Many studies have reported a variety of prognostic markers of OPC,
but they are either expensive or difficult to obtain. Therefore, we retrospectively studied the prognostic value
of circulating neutrophil count (CNC) in patients with OPC, with the aim of providing a theoretical basis for
further prognostic stratification. Methods: The clinicopathological data of 153 patients diagnosed with OPC from January 2010 to June 2017 were retrospectively analyzed. The CNC of each patient was measured before treatment. Then, the relationship between CNC and the clinicopathological characteristics of the patients was analyzed. The receiver operating characteristic (ROC) curve was used to calculate the cutoff value of CNC. The cox proportional hazards model was used to perform univariate and multivariate analysis of the relevant prognostic factors to determine the factors related to overall survival (OS) and progression-free survival (PFS). Results: The cutoff value for CNC was 4.48. Neutrophilia was significantly associated with disease stage, P16 status, and the type of therapy. In the univariate and multivariate analyses, CNC was found to be correlated with OS and PFS. Increased neutrophil count was predictive of poor $\mathrm{OS}(\mathrm{P}<0.001)$ and PFS $(\mathrm{P}=0.001)$. Neutrophil count was an independent risk factor for $\mathrm{OS}$ ( $\mathrm{HR}=2.09,95 \% \mathrm{CI}: 1.25-3.51, \mathrm{P}=0.005)$ and PFS (HR $=1.78,95 \% \mathrm{CI}: 1.10-2.88, \mathrm{P}=0.02$ ) in patients with OPC.

Conclusions: Pre-treatment CNC is an independent prognostic factor for OPC.

Keywords: Circulating neutrophil count (CNC); oropharyngeal cancer (OPC); prognosis; survival

Submitted Jul 13, 2020. Accepted for publication Sep 04, 2020.

doi: 10.21037/atm-20-5702

View this article at: http://dx.doi.org/10.21037/atm-20-5702

\section{Introduction}

Oropharyngeal cancer (OPC) is a malignant tumor that is associated with high rates of morbidity and mortality globally. Approximately two-thirds of patients with OPC are already at an advanced stage at the time of diagnosis, with localized lymph node or distant metastasis (1).

In recent years, a large number of studies have studied the correlation between various molecular biomarkers and 
the clinical outcomes of OPC patients. Molecular biological prognostic predictors can provide a wealth of prognostic and predictive information; however, their application in the clinical diagnosis and treatment of OPC is hindered by their high cost, lack of uniform standards, and limited regional availability $(2,3)$.

Systemic inflammatory factors have been reported to play essential roles in the progression and prognosis of different cancers, including neutrophil-to-lymphocyte ratio (NLR) and platelet-to-lymphocyte ratio (PLR), which are of great significance to the prognosis of OPC patients $(4,5)$.

Circulating neutrophil count (CNC) is proven as an independent prognostic indicator in various malignant tumors. Studies have shown that it holds significant prognostic value in advanced cancers, such as glioma, esophageal cancer, and lung cancer (6-9). However, no large-scale studies assessing the prognostic significance of CNC in OPC have been conducted to date. Therefore, we performed a retrospective study of the prognostic value of CNC in patients with OPC, with the aim of providing a theoretical basis for further prognostic stratification. We present the following article in accordance with the REMARK reporting checklist (available at http://dx.doi. org/10.21037/atm-20-5702).

\section{Methods}

\section{Patients}

The clinicopathological data of 153 newly diagnosed OPC patients who were treated in the Head and Neck Oncology Department of Tianjin Medical University Cancer Hospital between January, 2010, and June, 2017, were retrospectively analyzed. All procedures performed in this study involving human participants were in accordance with the Declaration of Helsinki (as revised in 2013). The study was approved by the ethics committee of the Tianjin Medical University Cancer Institute and Hospital. A signed written informed consent agreement was obtained from all patients before participation in the study. The inclusion criteria were as follows: (I) primary oropharyngeal squamous cell carcinoma confirmed by biopsy; (II) complete clinical and pathological data; and (III) complete blood cell count, including neutrophil count, obtained during the week before the start of treatment. The exclusion criteria were: (I) combined with other systemic malignancies; (II) incomplete clinicopathological data; (III) active inflammation or infection; or (IV) combined with immune disease.

\section{Treatment}

All patients received comprehensive treatment in the form of surgery or concurrent radiochemotherapy (with or without adjuvant chemotherapy), according to the $8^{\text {th }}$ edition of the National Comprehensive Cancer Network guidelines.

\section{Blood tests}

Blood samples were collected from all patients during the week before treatment. The patients' blood samples were stored in a tube treated with ethylenediamine tetraacetic acid. Then, routine peripheral blood cells, including lymphocytes, neutrophils, monocytes, eosinophils, basophils, and platelets, were analyzed with an automatic blood cell analyzer (Sysmex, Japan).

\section{Follow-up}

After treatment, all patients underwent a comprehensive physical examination every 3 to 6 months, and routine imaging examinations were performed at 3 and 9 months. If any signs of local progress or distant metastasis were detected during the follow-up, the relevant examinations were carried out at any time and the appropriate treatments were given. All patients were followed up for at least 3 years, or until death or disease progression or the study cutoff date (June 2020).

\section{Statistical analysis}

Patients with OPC were staged according to the $8^{\text {th }}$ edition of the American Joint Committee on Cancer staging system (patients with unknown p16 status were classified according to the p16-negative criteria). Subsequently, the patients were divided into two groups according to disease stage: the early group (stages I and II) and the late group (stages III and IV).

The study endpoints were overall survival (OS) and progression-free survival (PFS); OS was defined as death due to any cause, and PFS was defined as local progression, distant metastasis, or death due to disease progression.

Continuous variables were converted to categorical variables, and a chi-square test was used to compare categorical variables between groups. CNC served as the independent variable, and OS and PFS as endpoints. Receiver operating characteristic (ROC) curves were drawn, with the best cutoff point determined according to Youden's index, 


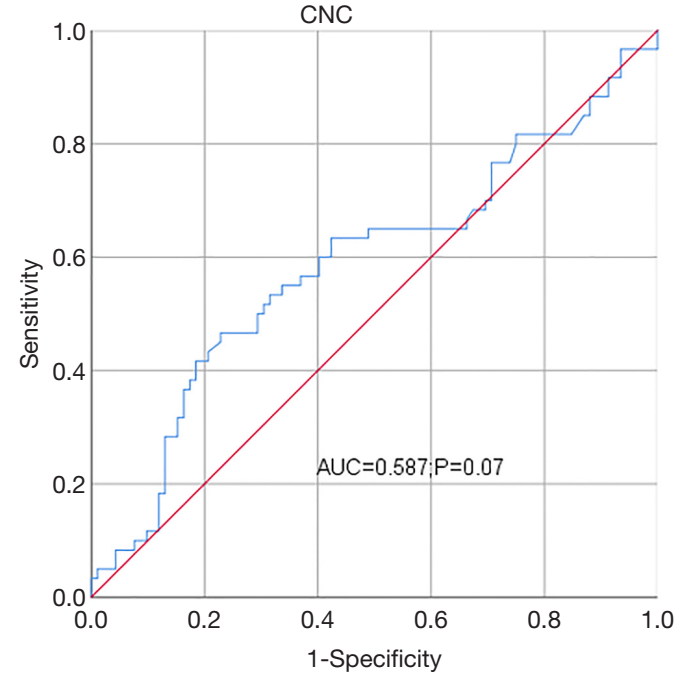

Figure 1 Receiver operating characteristic curve for circulating neutrophil count predicting overall survival. CNC, circulating neutrophil count.

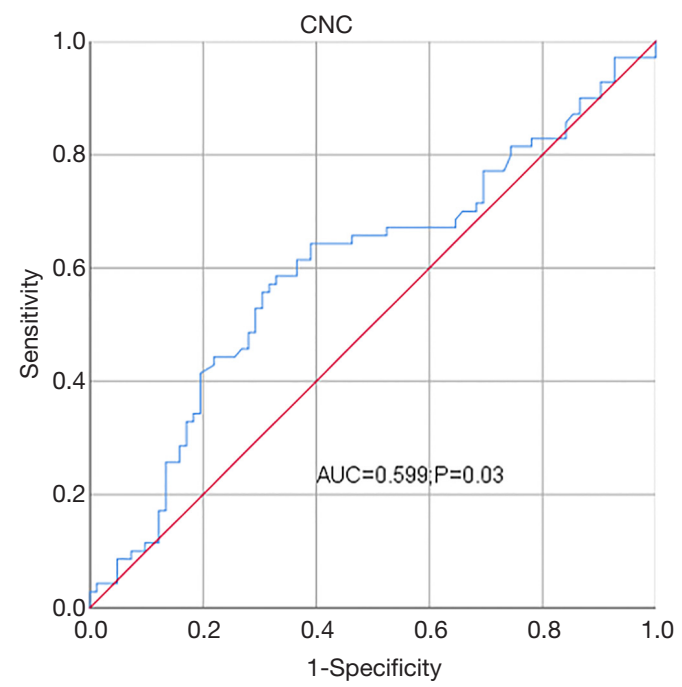

Figure 2 Receiver operating characteristic curve for circulating neutrophil count predicting progression-free survival. CNC, circulating neutrophil count.

and $\mathrm{CNC}$ was transformed into a classification variable. The Kaplan-Meier method was used to analyze survival curves, and the log-rank test was used to evaluate differences in survival rates between different $\mathrm{CNC}$ grades. Univariate and multivariate Cox proportional hazard models were used to identify prognostic factors associated with OS and PFS. A two-tailed $\mathrm{P}$ value of 0.05 was considered to be statistically significant. All statistical analyses were carried out using SPSSv26.0. (IBM Corporation, Armonk, NY, USA).

\section{Results}

\section{Patient characteristics}

A total of 153 patients with OPC were included in this study. The majority $(86.9 \%)$ of patients were male, and $59.5 \%$ were under the age of 60 years. Of the patients, $73.2 \%$ had a smoking history and $60.1 \%$ had a history of alcohol consumption. The primary tumor sites included the tonsil $(53.6 \%)$, the soft palate $(23.5 \%)$, and the base of the tongue (22.9\%). Most patients $(77.8 \%)$ were at an advanced stage (stage III or IV). Concurrent radiochemotherapy had been received by $71.9 \%$ of the patients, and the rest had received surgery. Of the patients, $11.8 \%$ were $\mathrm{P} 16$ positive and $19.6 \%$ were $\mathrm{P} 16$ negative, but the P16 status was unknown for the rest of the cohort.

The mean $\mathrm{CNC}$ was $(4.26 \pm 1.63) \times 10^{9} / \mathrm{L}$. The best cutoff point for CNC was determined for both OS (Figure 1) and PFS (Figure 2) according to Youden's index (cutoff value, $\left.4.48 \times 10^{9} / \mathrm{L}\right)$. CNC was then transformed into a classification variable according to this cutoff value $(\leq 4.48$, $\mathrm{n}=105 ;>4.48, \mathrm{n}=48$ ).

Table 1 shows the patients' baseline clinical characteristics according to $\mathrm{CNC}$. An increased $\mathrm{CNC}$ was related to disease stage $(\mathrm{P}=0.005), \mathrm{P} 16$ status $(\mathrm{P}=0.02)$, and the type of therapy $(\mathrm{P}=0.004)$. No significant differences existed among $\mathrm{CNC}$ groups for the other clinical characteristics

\section{Survival analysis}

The median follow-up time was 37 months (interquartile interval: $19-74$ months). A total of 60 (39.2\%) patients died and $71(46.4 \%)$ progressed. The 3- and 5-year OS rates for the 153 patients with OPC were $59.5 \%$ and $42.8 \%$, respectively. The 3- and 5-year PFS rates were $47.6 \%$ and $38.7 \%$, respectively.

Kaplan-Meier curve analysis revealed that the OS of patients with high CNC (>4.48) was significantly shorter than that of patients with low CNC $(<4.48)$ (log-rank $\mathrm{P}<0.001)$ (Figure 3). The 3 -year OS probability of patients in the high CNC group was $36.7 \%$, compared with $69.9 \%$ in the low CNC group. Also, patients with high CNC had significantly shorter PFS than patients with low CNC (logrank $\mathrm{P}=0.001$ ) (Figure 4). The 3 -year PFS rate was $28.7 \%$ and $56.5 \%$ in the high and low CNC groups, respectively. 
Table 1 Baseline clinical characteristics of the patients

\begin{tabular}{|c|c|c|c|c|}
\hline \multirow[b]{2}{*}{ Characteristics } & \multirow{2}{*}{$\begin{array}{c}\text { Total, n } \\
(\%)\end{array}$} & \multicolumn{2}{|c|}{$\mathrm{CNC}$} & \multirow[b]{2}{*}{$P$ value } \\
\hline & & $\begin{array}{c}\leq 4.48, \\
\mathrm{n}(\%)\end{array}$ & $\begin{array}{l}>4.48, \\
\mathrm{n}(\%)\end{array}$ & \\
\hline Total & 153 & 105 & 48 & \\
\hline Sex & & & & 0.09 \\
\hline Male & $133(86.9)$ & $88(83.8)$ & 45 (93.8) & \\
\hline Female & $20(13.1)$ & $17(16.2)$ & $3(6.2)$ & \\
\hline Age, years & & & & 0.85 \\
\hline$\leq 60$ & $91(59.5)$ & $63(60.0)$ & $28(58.3)$ & \\
\hline$>60$ & $62(40.5)$ & $42(40.0)$ & $20(41.7)$ & \\
\hline Smoking & & & & 0.46 \\
\hline Never & $41(26.8)$ & $30(28.6)$ & $11(22.9)$ & \\
\hline Formerly or currently & $112(73.2)$ & 75 (71.4) & $37(77.1)$ & \\
\hline Alcohol consumption & & & & 0.96 \\
\hline Never & $61(39.9)$ & $42(40.0)$ & $19(39.6)$ & \\
\hline Formerly or currently & $92(60.1)$ & $63(60.0)$ & $29(60.4)$ & \\
\hline Primary tumor & & & & 0.08 \\
\hline Tonsil & $82(53.6)$ & $50(47.6)$ & $32(66.7)$ & \\
\hline Base of tongue & 35 (22.9) & $28(26.7)$ & 7 (14.6) & \\
\hline Soft palate & $36(23.5)$ & $27(25.7)$ & $9(18.8)$ & \\
\hline Disease stage & & & & 0.005 \\
\hline $\mathrm{I} / \mathrm{II}$ & 34 (22.2) & $30(28.6)$ & $4(8.3)$ & \\
\hline III/IV & $119(77.8)$ & $75(71.4)$ & $44(91.7)$ & \\
\hline P16 status & & & & 0.02 \\
\hline Positive & $18(11.8)$ & $17(16.2)$ & $1(2.1)$ & \\
\hline Negative & $30(19.6)$ & $22(21.0)$ & $8(16.7)$ & \\
\hline Unknown & $105(68.6)$ & $66(62.8)$ & $39(81.3)$ & \\
\hline Type of therapy & & & & 0.004 \\
\hline Surgery & $43(28.1)$ & $37(35.2)$ & $6(12.5)$ & \\
\hline Chemo-radiotherapy & $110(71.9)$ & $68(64.8)$ & $42(87.5)$ & \\
\hline
\end{tabular}

CNC, circulating neutrophil count.

In Table 2 shows the significant correlation between OS and disease stage $(\mathrm{P}<0.001)$ and $\mathrm{CNC}(\mathrm{P}<0.001)$ after univariate analysis. Advanced disease $(\mathrm{P}<0.001)$, higher CNC $(\mathrm{P}=0.001)$, and alcohol consumption $(\mathrm{P}=0.04)$ were significantly correlated with PFS.

Subsequently, the above three variables were included

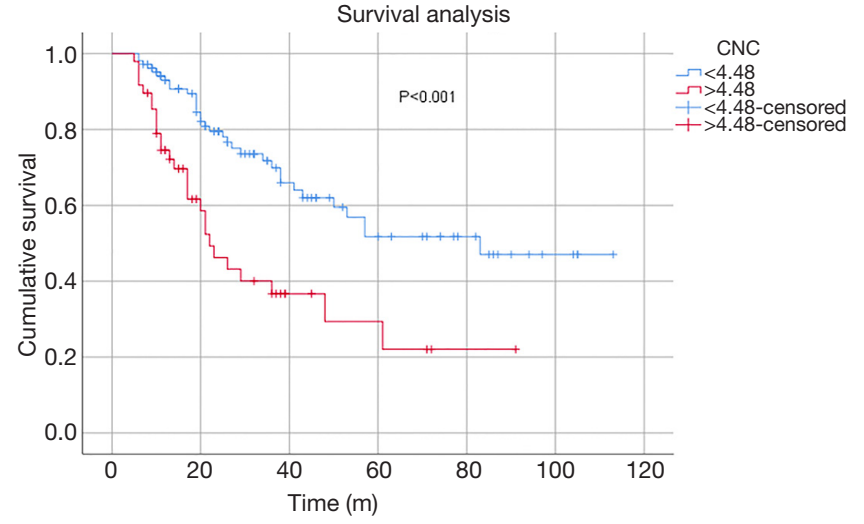

Figure 3 Kaplan-Meier curve of cumulative overall survival in 153 patients with oropharyngeal cancer according to circulating neutrophil count classification. CNC, circulating neutrophil count.

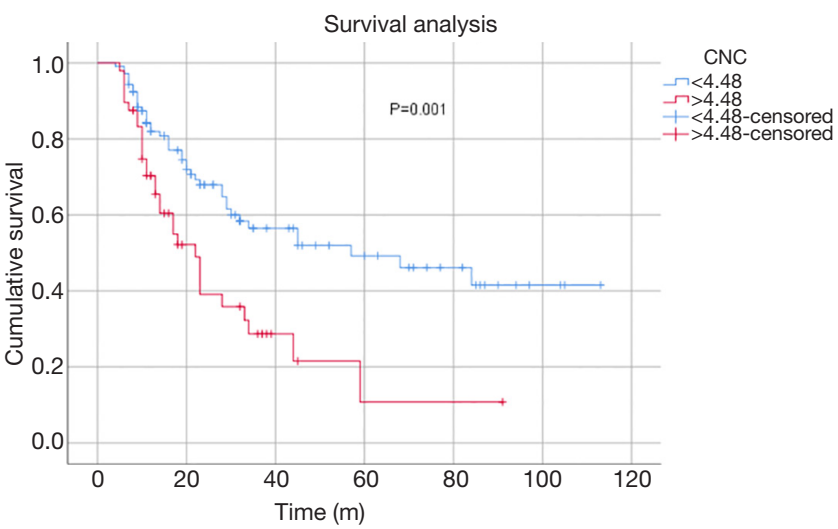

Figure 4 Kaplan-Meier curve of cumulative progression-free survival in 153 patients with oropharyngeal cancer according to circulating neutrophil count classification. CNC, circulating neutrophil count.

in the multivariate analysis. Multivariate Cox proportional hazard models were used to determine the independent prognostic factors of OS and PFS (Table 3).

Multivariate analysis revealed that $\mathrm{CNC}$ was an independent influencing factor of OS (HR $=2.09,95 \%$ CI: $1.25-3.51$, $\mathrm{P}=0.005)$ and PFS (HR $=1.78,95 \%$ CI: $1.10-2.88, \mathrm{P}=0.02$ ). At the same time, disease stage was also an independent factor affecting OS (HR $=5.50,95 \% \mathrm{CI}: 1.95-15.56, \mathrm{P}=0.001)$ and PFS (HR $=3.93$, 95\% CI: $1.75-8.83, \mathrm{P}=0.001)$.

\section{Discussion}

In this study, we retrospectively analyzed the clinicopathological 
Table 2 Univariate Cox regression analysis for overall survival and progression-free survival in patients with oropharyngeal cancer

\begin{tabular}{|c|c|c|c|c|c|c|}
\hline Variables & \multicolumn{3}{|c|}{ Overall survival } & \multicolumn{3}{|c|}{ Progression-free survival } \\
\hline Sex & 0.86 & $0.34-2.15$ & 0.74 & 0.61 & $0.25-1.52$ & 0.29 \\
\hline Age & 1.23 & $0.74-2.05$ & 0.42 & 1.09 & $0.68-1.74$ & 0.72 \\
\hline Smoker & 1.14 & $0.62-2.11$ & 0.67 & 1.31 & $0.74-2.31$ & 0.36 \\
\hline Primary tumor & 1.06 & $0.91-1.22$ & 0.48 & 1.08 & $0.94-1.23$ & 0.29 \\
\hline Disease stage & 6.90 & $2.48-19.24$ & $<0.001$ & 4.72 & $2.13-10.46$ & $<0.001$ \\
\hline P16 status & 1.16 & $0.80-1.70$ & 0.44 & 1.06 & $0.76-1.46$ & 0.75 \\
\hline Type of therapy & 1.30 & $0.96-1.77$ & 0.09 & 1.17 & $0.89-1.54$ & 0.27 \\
\hline
\end{tabular}

CNC, circulating neutrophil count.

Table 3 Multivariate cox regression analysis for overall survival and progression-free survival in patients with oropharyngeal cancer

\begin{tabular}{|c|c|c|c|c|c|c|}
\hline Variables & \multicolumn{3}{|c|}{ Overall survival } & \multicolumn{3}{|c|}{ Progression-free survival } \\
\hline Alcohol consumption & 1.40 & $0.80-2.44$ & 0.24 & 1.53 & $0.92-2.56$ & 0.10 \\
\hline Disease stage & 5.50 & $1.95-15.56$ & 0.001 & 3.93 & $1.75-8.83$ & 0.001 \\
\hline CNC & 2.09 & $1.25-3.51$ & 0.005 & 1.78 & $1.10-2.88$ & 0.02 \\
\hline
\end{tabular}

CNC, circulating neutrophil count.

data of a group of OPC patients to determine the prognostic value of pre-treatment $\mathrm{CNC}$ in OPC. We found that a higher pre-treatment $\mathrm{CNC}$ was independently associated with poor OS and PFS in OPC patients. Our findings suggest that pretreatment $\mathrm{CNC}$ may be a new indicator for the prognostic stratification of OPC patients.

Recent studies have shown that various pro-inflammatory mediators are associated with tumor progression at different stages of head and neck squamous cell carcinoma (HNSCC), and contribute to cancer cell proliferation, neovascularization, distant metastasis, and drug resistance. In previous studies, complex inflammation was demonstrated to play an essential role in the progression and metastasis of HNSCC (10-13).

Some studies have reported that the CNC of tumor patients was significantly higher than that of individuals without cancer, with different effects $(14,15)$. Basic studies have shown that tumor cells may stimulate the release of neutrophils from the bone marrow by releasing various cytokines, leading to an increase in $\mathrm{CNC}$, after which neutrophils are recruited to the primary tumor site $(16,17)$.
In previous studies, elevated $\mathrm{CNC}$ has been shown to be involved in tumor angiogenesis and to induce tumor cells to develop resistance to anti-vascular endothelial growth factor (VEGF) therapy (18). In recent years, many experts and scholars have discussed the relationship between $\mathrm{CNC}$ and the prognosis of various cancers, including lung cancer, gastric cancer, colorectal cancer, and esophageal cancer $(7,8,19,20)$.

CNC has been shown to play an important role in the prognosis of patients with head and neck cancer, including OPC, in several studies. For instance, Su et al. reported that leukopenia and neutrophilia were independent factors of poor prognosis in patients with nasopharyngeal carcinoma (21). Furthermore, Valero et al. revealed that high pre-treatment counts of peripheral blood neutrophils and/or monocytes are an independent predictor of poor prognosis in patients with HNSCC (22). Huang et al.'s retrospective analysis of a large cohort of OPC patients discovered that high CNC independently predicted worse OS and RFS in patients with human papillomavirus 
(HPV) (+) OPC. However, CNC cannot be used to predict the prognosis of HPV (-) patients (23). Huang et al. use the median of $\mathrm{CNC}\left(4.7 \times 10^{9} / \mathrm{L}\right)$ as the grouping standard, and our study used ROC curves to calculate the Jordan index as the grouping basis of $\mathrm{CNC}\left(4.48 \times 10^{9} / \mathrm{L}\right)$. Although the grouping methods employed in the studies differ, the cutoff values are very close. Huang et al.'s study suggests that CNC can only predict the prognosis of HPV (+) OPC patients, which contrasts with our finding that $\mathrm{CNC}$ can be used to predict the prognosis of all OPC patients. This difference may be attributable to the excessive proportion of patients with unknown P16 status in our data. In future research, we will expand the sample size and determine the status of $\mathrm{HPV}$ infection in patients. Our current findings encourage us to pay attention to OPC patients with high CNC during clinical diagnosis and treatment, to administer antiinflammatory treatment if necessary, and to observe whether the prognosis can be improved after $\mathrm{CNC}$ degradation.

Our research also has the following limitations. This study is a retrospective study, which may have led to the exclusion of a large number of patients with incomplete case data, resulting in inevitable selection bias. Moreover, our study did not take into account the unknown confounding factors that could potentially impact the CNC. The sample size of this study was also small, which contributed to a relatively small area under the ROC curve. In order to more accurately understand the guiding significance of $\mathrm{CNC}$ on the prognosis of patients with OPC, a well-designed prospective study with a sufficient sample size is needed in the future.

\section{Conclusions}

Our results showed that a high pretreatment CNC was independently related to worse OS and PFS in patients with OPC. As an economical pre-treatment blood biomarker, the combined application of CNC and the TNM clinical staging system can obtain more accurate prognostic information and provide a theoretical basis for the development of prognostic stratification of OPC patients in the future.

\section{Acknowledgments}

Funding: None.

\section{Footnote}

Reporting Checklist: The authors have completed the
REMARK reporting checklist. Available at http://dx.doi. org/10.21037/atm-20-5702

Data Sharing Statement: Available at http://dx.doi. org/10.21037/atm-20-5702

Conflicts of Interest: All authors have completed the ICMJE uniform disclosure form (available at http://dx.doi. org/10.21037/atm-20-5702). The authors have no conflicts of interest to declare.

Ethical Statement: The authors are accountable for all aspects of the work in ensuring that questions related to the accuracy or integrity of any part of the work are appropriately investigated and resolved. All procedures performed in this study involving human participants were in accordance with the Declaration of Helsinki (as revised in 2013). The study was approved by the ethics committee of the Tianjin Medical University Cancer Institute and Hospital. A signed written informed consent agreement was obtained from all patients before participation in the study.

Open Access Statement: This is an Open Access article distributed in accordance with the Creative Commons Attribution-NonCommercial-NoDerivs 4.0 International License (CC BY-NC-ND 4.0), which permits the noncommercial replication and distribution of the article with the strict proviso that no changes or edits are made and the original work is properly cited (including links to both the formal publication through the relevant DOI and the license). See: https://creativecommons.org/licenses/by-nc-nd/4.0/.

\section{References}

1. Mallen-St Clair J, Ho AS. American Joint Committee on Cancer 8th edition staging-an improvement in prognostication in HPV-associated oropharyngeal cancer? Ann Transl Med 2019;7:S10.

2. Misawa K, Mima M, Satoshi Y, et al. Neuropeptide receptor genes GHSR and NMUR1 are candidate epigenetic biomarkers and predictors for surgically treated patients with oropharyngeal cancer. Sci Rep 2020;10:1007.

3. Sano D, Oridate N. Pretreatment prognostic factor for patients with human papillomavirus related oropharyngeal cancer. Transl Cancer Res 2019;8:354-6.

4. Ng SP, Bahig H, Jethanandani A, et al. Prognostic Significance Of Pre-Treatment Neutrophil-ToLymphocyte Ratio In Patients with Oropharyngeal Cancer 
Treated with Radiotherapy. Int J Radiat Oncol Biol Phys 2019;105:E426-7.

5. Martucci F, Martucci F, Cima S, et al. Neutrophil to lymphocyte ratio (NLR) and platelet to lymphocyte ratio (PLR) as prognostic markers in locally advanced squamous cell oropharyngeal cancer: a retrospective, observational, multicenter experience. Strahlentherapie Und Onkologie 2019;195:1143.

6. Wang Z, Zhong L, Li G, et al. Pre-treatment neutrophils count as a prognostic marker to predict chemotherapeutic response and survival outcomes in glioma: a single-center analysis of 288 cases. Am J Transl Res 2020;12:90-104.

7. Luo HS, Xu HY, Du ZS, et al. Prognostic Significance of Baseline Neutrophil Count and Lactate Dehydrogenase Level in Patients With Esophageal Squamous Cell Cancer Treated With Radiotherapy. Front Oncol 2020;10:430.

8. Wang Z, Zhan P, Lv Y, et al. Prognostic role of pretreatment neutrophil-to-lymphocyte ratio in non-small cell lung cancer patients treated with systemic therapy: a meta-analysis. Transl Lung Cancer Res 2019;8:214-26.

9. Mandaliya H, Jones M, Oldmeadow C, et al. Prognostic biomarkers in stage IV non-small cell lung cancer (NSCLC): neutrophil to lymphocyte ratio (NLR), lymphocyte to monocyte ratio (LMR), platelet to lymphocyte ratio (PLR) and advanced lung cancer inflammation index (ALI). Transl Lung Cancer Res 2019;8:886-94.

10. Dong GW, Do NY, Lim SC, et al. Relation between proinflammatory mediators and epithelial-mesenchymal transition in head and neck squamous cell carcinoma. Exp Ther Med 2010;1:885-91.

11. Koontongkaew $\mathrm{S}$. The tumor microenvironment contribution to development, growth, invasion and metastasis of head and neck squamous cell carcinomas. J Cancer 2013;4:66-83.

12. Bonomi M, Patsias A, Posner M, et al. The role of inflammation in head and neck cancer. Adv Exp Med Biol 2014;816:107-27.

13. Diao P, Wu Y, Ge H, et al. Preoperative circulating platelet, neutrophil, and lymphocyte counts predict survival in oral cancer. Oral Dis 2019;25:1057-66.

14. Trellakis S, Farjah H, Bruderek K, et al. Peripheral blood neutrophil granulocytes from patients with head and neck squamous cell carcinoma functionally differ from their counterparts in healthy donors. Int J Immunopathol
Pharmacol 2011;24:683-93.

15. Fernandes PC Jr, Garcia CB, Micheli DC, et al. Circulating neutrophils may play a role in the host response in cervical cancer.Int J Gynecol Cancer 2007;17:1068-74.

16. Dumitru CA, Lang S, Brandau S. Modulation of neutrophil granulocytes in the tumor microenvironment: mechanisms and consequences for tumor progression. Semin Cancer Biol 2013;23:141-8.

17. Tazzyman S, Niaz H, Murdoch C. Neutrophil-mediated tumour angiogenesis: subversion of immune responses to promote tumour growth. Semin Cancer Biol 2013;23:149-58.

18. Zhang H, Shang X, Ren P, et al. The predictive value of a preoperative systemic immune-inflammation index and prognostic nutritional index in patients with esophageal squamous cell carcinoma. J Cell Physiol 2019;234:1794-802.

19. Chen Z, Chen W, Wang J, et al. Pretreated baseline neutrophil count and chemotherapy-induced neutropenia may be conveniently available as prognostic biomarkers in advanced gastric cancer. Intern Med J 2015;45:854-9.

20. Watt DG, Martin JC, Park JH, et al. Neutrophil count is the most important prognostic component of the differential white cell count in patients undergoing elective surgery for colorectal cancer. Am J Surg 2015;210:24-30.

21. Su Z, Mao YP, Ouyang PY, et al. Initial Hyperleukocytosis and Neutrophilia in Nasopharyngeal Carcinoma: Incidence and Prognostic Impact. PLoS One 2015;10:e136752.

22. Valero C, Pardo L, López M, et al. Pretreatment count of peripheral neutrophils, monocytes, and lymphocytes as independent prognostic factor in patients with head and neck cancer. Head Neck 2017;39:219-26.

23. Huang SH, Waldron JN, Milosevic M, et al. Prognostic value of pretreatment circulating neutrophils, monocytes, and lymphocytes in oropharyngeal cancer stratified by human papillomavirus status. Cancer 2015;121:545-55.

(English Language Editor: J. Reynolds)

Cite this article as: Li L, Liu G, Lu H, Jin K, Zhai X, Zhou M, Duan Y, Yue K, Wu Y, Wang X. Pre-treatment circulating neutrophil count is an independent prognostic factor in oropharyngeal cancer. Ann Transl Med 2020;8(18):1135. doi: 10.21037/atm-20-5702 